\title{
Aversive thresholds for subcutaneous electrical stimulation in the cat
}

\author{
Dennis D. Kelly and Murray Glusman \\ COLUMBIA UNIVERSITY AND NEW YORK STATE PSYCHIATRIC INSTITUTE
}

\begin{abstract}
Reliable, long-term aversive thresholds were obtained in unrestrained cats with a titration schedule. These thresholds, marked by low variability, were found comparable to those obtained with a reset-to-zero procedure. A subcutaneous implant technique permitted individual determination of thresholds for homologous body areas and their selective alteration by a local anesthetic.
\end{abstract}

\section{Problem}

Weiss \& Laties (1958) apply the term "titration" to the procedure in which an animal's response reduces by a given amount the intensity of some noxious stimulus. This stimulus is applied continuously, and if the animal does not respond, its intensity is programmed to increase as a step-like function. If the experimenter focuses his attention upon the level of the stimulus "tolerated" by the animal, then titration can be viewed as a sensory threshold technique. Psychophysically, it represents a continuous method of limits in which the stimulus is controlled, and thereby reported upon, by the subject. Further, if a threshold is taken to be simply some statistical boundary separating stimulus values that elicit one response (bar press) from the stimuli that elicit a different response (no bar press), then clearly titration is a method which displays all of the operations which define the concept of "threshold." 'A major problem is to identify the sensory referent for this threshold.

To indicate thresholds precisely, titration must first produce a baseline level low in variability, replicable from day to day, and resistant to adaptational "drifting.' Recent approaches (Boren \& Malis, 1961; Weiss \& Laties, 1962; Weitzman \& Ross, 1962) which have met these demands have also severely restricted the animal, and thus were not applicable to our design which demanded an unrestrained cat.

\section{Method}

Much of the behavioral variability originally' noted with titration probably reflects fluctuation of certain critical, but uncontrolled, stimulus parameters. We have found that a stimulus defined in terms of a constant AC current produces a close approximation to a constant behavioral effect-provided current density and the site of stimulation are kept constant. To control the electrode surface area, and thereby current density, two stainless steel screws imbedded $5 / 8$ in apart in a polyethylene disk were anchored subcutaneously in each flank of a cat. From these electrodes subcutaneous wires were run in polyethylene tubing to a connector mounted on the skull. Continuous 100 cps biphasic stimulation could then be applied unilaterally to either flank, or bilaterally by using one electrode from each side.

Preliminary to threshold testing, seven cats received $15 \mathrm{hr}$. training on a grid-shock escape task. Each cat attained a stable response rate showing few extras and little bursting. At this time, the noxious stimulus was changed from foot shock to subcutaneous flank shock, and the cat transferred to a titration schedule. Shock intensity ranged from 0 to 2 ma in 25 equal interval steps. Time between successive shock increments was constant at $4 \mathrm{sec}$; however, in some cases this interval was made variable with no systematic effects.

\section{Results and Discussion}

Figure 1A shows the first $40 \mathrm{~min}$. of a typical animal's responding immediately after transfer from escape to

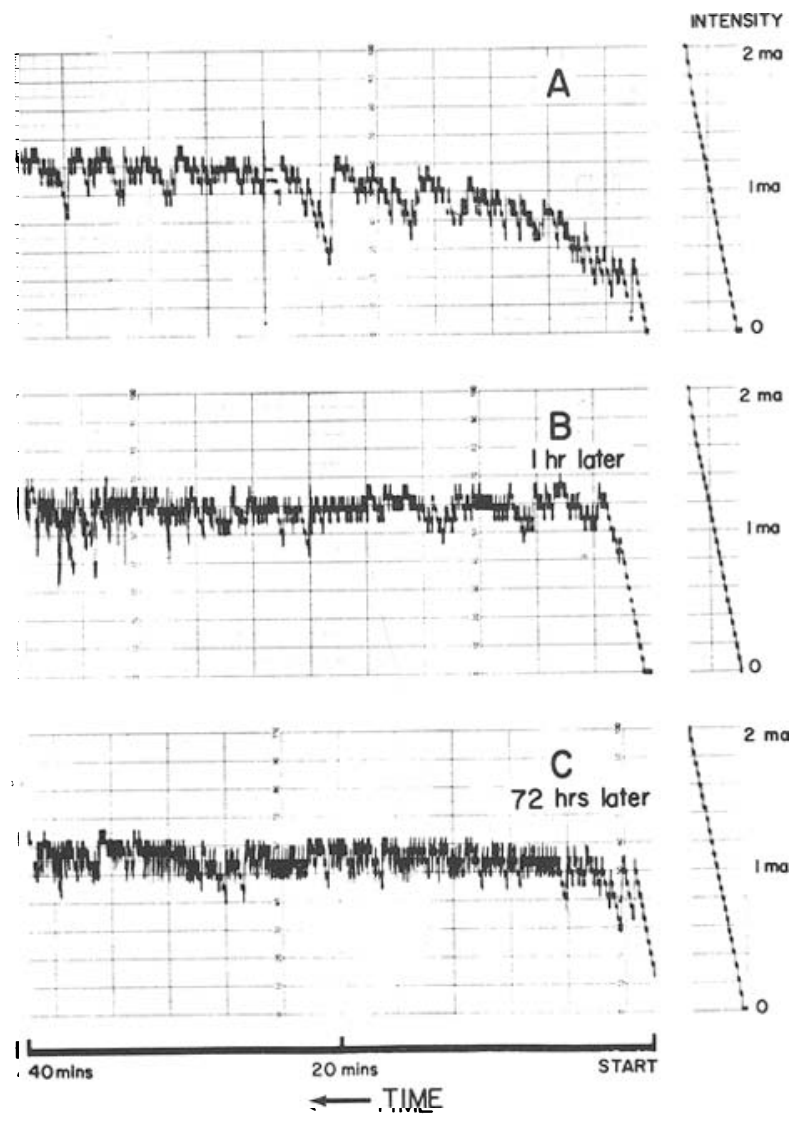

Fig. 1. Titration responding in the cat. A: Initial exposure to schedule; first $40 \mathrm{~min}$. of a $3-\mathrm{hr}$. session. B: Resumption of titration following 5-min. rest period midway through same session. C: Initial $40 \mathrm{~min}$. on third day of experiment. Records read from right to left. 
titration. Note the low variability and the fast assumption of a clearly definable baseline of $1.2 \mathrm{ma}$. This level undergoes little adaptation even after $2 \mathrm{hr}$. of continuous stimulation (compare Fig. 1A to 1B). In fact, this threshold is recoverable from one day to the next, even for the first $40 \mathrm{~min}$. of each session (compare Fig. 1A to 1C). After prolonged exposure (usually about 35-session $\mathrm{hr}$.), an increase in variability is noticed. This effect is highly skewed with the cats now responding more at lower levels of shock than previously, while still never allowing the stimulus to rise above the original titrated level. Such variability, usually interpreted as the acquisition of a classical or Sidman-type avoidance response (since both contingencies are present in titration), is normally associated with the initiation of bursting (several bar presses in rapid succession) rather than an evenly spaced increase in rate. Such bursts can be counteracted, and the low-variability baseline recovered, by introducing a small drl contingency so that any bar press following the previous bar press by less than .75 sec. is ineffective in reducing the shock. On this schedule our cats have demonstrated consistent thresholds for 16 weeks or longer.

In a related procedure, initially used by Mowrer (1940), each response reset directly to zero the intensity of a gradually-built-up shock. Applied to titration, this seemingly small modification of the response contingency radically alters the probable temporal programming of the aversive stimulus. However, like titration, the reset-to-zero procedure displays all the necessary logical operations for obtaining a threshold. Therefore, a comparison of thresholds obtained by these two procedures provides opportunity for a cross-test validation of titration. The results of such a test are shown in Fig. 2 for records obtained in successive 35-min. sessions. The evident comparability of the levels traced by these methods strongly suggests that the animal's responding has come under the control of a sensory event common to both.

It may be unjustified to conclude that "pain," or unconditioned aversive stimulation, is the controlling sensory event. As Boren \& Malis (1961) have noted, titration is undoubtedly complicated by discriminated avoidance behavior with low shock levels serving as warning stimuli for higher ones. Yet, we have found it possible to selectively elevate by procaine injection the threshold of one side of the cat's body without affecting those for the opposite, control, side. This effect mimics the sensitivity of titration to a general analgesic (Weitzman \& Ross, 1962), but eliminates the possibility of generalized, non-stimulus, effects of the drug shifting thresholds by slowing response rates. Still, it may be argued that any aversive properties conditioned to the low-level stimuli, if not themselves affected by the analgesic, extinguished with the removal of the primary aversive stimuli maintaining them.
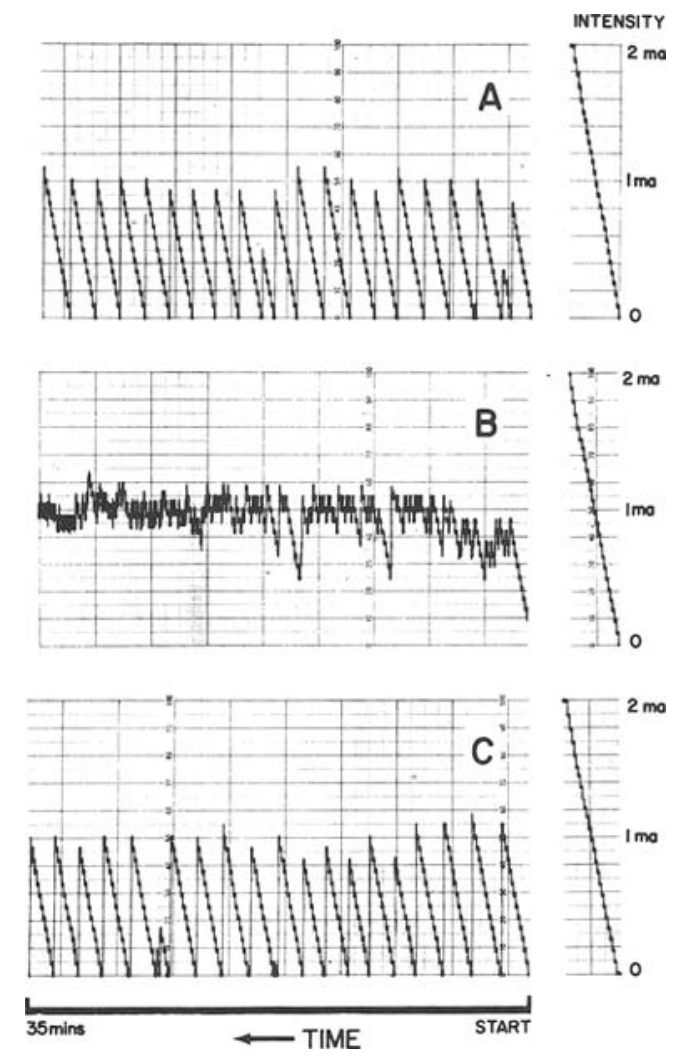

Fig. 2. Aversive thresholds obtained under two schedules. A \& C: Each response resets shock intensity back to zero. B: Titration procedure. A, B, and C are successive records on same day separated by $5-$ min. rest periods.

\section{References}

BOREN, J. J., \& MALIS, J. L. Determining thresholds of aversive brain stimulation. Amer. J. Physiol., 1961, 201, 429-433.

MOWRER, O. H. An experimental analogue of "regression" with incidental observations on "reaction formation." J. abnorm. soc. Psychol., 1940, 35, 56-87.

WEISS, B., \& LATIES, V. Fractional escape and avoidance on a. titration schedule. Science, 1958, 128, 1575-1576.

WEISS, B., \& LATIES, V. A foot electrode for monkeys. J.exp. Anal. Beh., 1962, 5, 535-536.

WEITZMAN, E. D., \& ROSS, G. A behavioral method for the study of pain perception in the monkey. Neurology; 1962, 12, 264-272.

\section{Note}

1. This research was supported by Grants $\mathrm{MH}-03660$ and FR-05248-01 from the U. S. Public Health Service. 